\title{
Fuzzy based Stochastic Algorithms for Multi-Objective Reactive Power Optimization including FACTS Devices
}

\author{
D. Silas Stephen ${ }^{1}$, P. Somasundaram ${ }^{2}$ \\ 1Research scholar, Department of Electrical and Electronics Engineering, CEG, \\ Anna University, Chennai, India-600 025 \\ 2Assistant Professor, Department of Electrical and Electronics Engineering, CEG, \\ Anna University, Chennai, India-600 025
}

\begin{abstract}
Fuzzy based stochastic algorithms for solving Multi-objective Reactive Power Optimization (MORPO) problem including FACTS devices is presented in this paper. The Multi-Objective Reactive Power Optimization problem is formulated as a nonlinear constrained multi-objective optimization problem where the active power, voltage deviation and investment cost has to be minimized simultaneously. Fuzzy logic strategy incorporated with Evolutionary Programming (EP), Tabu search (TS) algorithms and Particle Swarm Optimization (PSO) has been proposed to handle the problem as a true multi-objective problem. The proposed algorithm has been used to solve the MORP problem with and without FACTS devices namely STATCOM, TCSC and UPFC.IEEE 30-bus system is used as a test system. The simulation results are promising and show the effectiveness and robustness of the proposed approach.
\end{abstract}

Keywords: Multi-objective Reactive Power Optimization; Evolutionary Programming; Tabu Search; Particle Swarm Optimization; Fuzzy Logic; FACTS

\section{Introduction}

The Multi-Objective Reactive Power Optimization (MORPO) problem is a complex problem in which several parameters like Power loss, Voltage deviation, investment cost of the devices and L-index have to be independently and simultaneously optimized. The Decision making process in MORP is so complex and the Decision Maker (DM) is faced with multiple objectives. The multiple objectives in a problem gives out a set of optimal solutions, known as Pareto Optimal solutions. To solve the MORP sufficient information are required. The DM has to choose an optimal solution by generating as many Pareto solutions as possible from which the optimal solution can be chosen $[1,2]$.

In power system, MO problems are solved by converting into a Single Objective by various methods like Goal-attainment method [3], weighted sum [4], $\xi$-constraint approach, Normalization method, Weight-norm method [6], fuzzy method [7] and projection method [5]. Now days a number of Multi-Objective Evolutionary Algorithms (MOEA) have been proposed which has the ability of find multiple Pareto-optimal solutions in one single run. Evolutionary algorithms (EA) inherently explore a set of possible solutions simultaneously [6]. Genetic algorithm [7,8], Evolutionary programming, Fast Evolutionary Programming, Particle Swarm Optimization and Simulated Annealing, Niched Pareto Genetic Algorithm [9,10], Non-dominated Sorting Genetic Algorithm [14], Strength Pareto Evolutionary Algorithm [11] and SPEA2 (Improving Strength Pareto Evolutionary Algorithm) [12] and Differential Evolution [13] have also been used for solving MO problems.

The MORP problem can be solved by using the Evolutionary programming (EP) algorithm [14], Tabu Search (TS) algorithm and Particle Swarm Optimization (PSO) [15] algorithm. The

Received: April $12^{\text {th }}, 2012$. Accepted: June $14^{\text {th }}, 2012$ 
performance of EP is better for MO problems but the major drawback is its inconsistent convergence and computation time [16]. The TS algorithm has the ability to avoid entrapments in local optimum but it requires larger computation time. The PSO algorithm has faster convergence and simplicity but it requires more number of iteration to converge. Hence there is a need to accelerate the convergence of EP, TS and PSO algorithms and thereby reducing the computation time. In the recent years there has been an increase in the application of Fuzzy Logic strategy, as it is a non-linear mapping of input data vector into scalar output which requires appropriate but partial information of criteria only [18-19]. So in this paper Fuzzy Logic has been incorporated with the EP, TS and PSO algorithms in order to minimize the convergence and computation time. In EP the mutation process is incorporated with Fuzzy Logic which leads to an improved technique called as Fuzzy Mutated Evolutionary Programming (FMEP).In TS algorithm Fuzzy logic has been implemented [17] in the mutation and recombination process of TS algorithm and it is termed as Fuzzy Guided Tabu Search (FGTS). Fuzzy Logic strategy has been incorporated to adjust the parameters of PSO and termed as Fuzzified PSO.

FACTS devices are used to regulate the real and reactive power by their swift control characteristics and compensating ability. A coordination between FACTS devices and the conventional power system control devices makes the power system to operate in a more secure and economical way $[18,19]$. FACTS controllers reduces the systems losses and improves stability without any change topological changes or generation rescheduling. Therefore, it becomes necessary to incorporate the FACTS devices like SVC, TCSC and UPFC with the power system.

In this paper a Multi Objective Reactive Power Optimization (MORPO) problem is considered which has a linear combination of several factors such as: investment cost, Voltage deviation and transmission losses, subject to operational constraints such as reliability and voltage profile. This problem is complex as it has multiple objectives to be minimized simultaneously. The MORPO in coordination with FACTS devices is solved with the FMEP, FGTS and FPSO. IEEE 30-bus system is used as the test system as it has more control variables and provides result for the comparison for the proposed methods. The simulation results prove the ability of the proposed method in generating well distributed pareto-optimal solutions of the MORPO problem with better convergence and minimal computation time.

\title{
2. Multi-objective Reactive Power Optimization Problem
}

MORPO problem is a linear combination of three objective functions which are to be simultaneously optimized namely active power loss (F1), Maximum voltage Deviation (F2) and Cost of the Compensating devices (F3).

\author{
A. Active power loss: \\ $\mathrm{F} 1=\mathrm{Pg}-\mathrm{Pd}$ \\ The total power loss is always positive. $\mathrm{F} 1 \geq 0$. \\ F1 The total transmission active losses of the power system in MW. \\ $\mathrm{Pg} \quad$ The total active power generated in MW \\ Pd The total load of the system in MW.
}

B. Maximum voltage deviation:

$$
\mathrm{F}_{2}=\max \left(\mathrm{V}_{\mathrm{i}}-\mathrm{V}_{\mathrm{i}}^{*}\right)=\left\|\mathrm{V}_{\mathrm{i}}-\mathrm{V}_{\mathrm{i}}^{*}\right\|_{\infty} \geq 0
$$

Where,

F2 The maximum voltage deviation from the desired value (P.U).

$\mathrm{V} \varepsilon \mathrm{R}^{\mathrm{n}} \quad$ The voltage vector (unknown).

$\mathrm{V}^{*} \varepsilon \mathrm{R}^{\mathrm{n}} \quad$ The desired voltage vector. 
C. Cost of the Compensating devices:

$$
\begin{aligned}
& \mathrm{F}_{3}=\sum_{\mathrm{i}=1}^{\mathrm{n}} \mathrm{k}\left|\mathrm{B}_{\mathrm{i}}\right| \\
& \mathrm{k}=\left\{\begin{array}{r}
\alpha \text { if } 0 \leq \mathrm{B}_{\mathrm{i}} \leq \mathrm{B}_{\mathrm{m}} \\
\beta \text { if }-\mathrm{B}_{\mathrm{m}} \leq \mathrm{B}_{\mathrm{i}} \leq \mathrm{B}_{\mathrm{o}}
\end{array}\right. \\
& \mathrm{F} 3 \leq \mathrm{F}_{3}^{\max }
\end{aligned}
$$

$\mathrm{Bi}$

Compensation at bus bar i in MVAr

Bm Maximum amount of compensation in MVAr allowed at a single bus bar.
A Cost per MVAr of a capacitor bank
B Cost per MVAr of a reactor bank and $\mathrm{n}$ is the number of bus bars in the electric power system.
$\mathrm{F}_{3}^{\max } \quad$ The maximum amount available for investment

The cost of the FACTS devices according to Siemens AG database is governed by the equations:

$$
\begin{aligned}
& C_{S V C}=0.0003 S^{2}-0.3051 S+127.38 \\
& C_{T C S C}=0.0015 S^{2}-0.713 S+153.75 \\
& C_{U P F C}=0.0003 S^{2}-0.32691 S+188.22
\end{aligned}
$$

Where, $C_{S V C}, C_{T C S C}$ and $C_{U P F C}$ are the cost functions of SVC, TCSC and UPFC respectively in US\$/KVAr and $\mathrm{S}$ is the operating range of the FACTS controllers in MVAr. The problem is summarized as

$$
\mathrm{F}=\left[\mathrm{P}_{\mathrm{g}}-\mathrm{P}_{\mathrm{d}}\left\|\mathrm{V}-\mathrm{V}^{*}\right\|_{\infty} \sum_{\mathrm{i}=1}^{\mathrm{n}} \mathrm{k}\left|\mathrm{B}_{\mathrm{i}}\right|\right]
$$

Subject to the constraints

Load flow constraints:

$$
\begin{array}{ll}
P_{G i}-P_{D i}=V_{i} \sum V_{j}\left(G_{i j} \cos \theta_{i j}+B_{i j} \sin \theta_{i j}\right) & \mathrm{i} \in N_{B} \\
Q_{G i}-Q_{D i}=V_{i} \sum V_{j}\left(G_{i j} \sin \theta_{i j}-B_{i j} \cos \theta_{i j}\right) & \mathrm{i} \in N_{P Q}
\end{array}
$$

Voltage constraints: $V_{i}^{\min } \leq V_{i} \leq V_{i}^{\max } \quad i \in N_{B}$

Generator reactive power generation limit: $Q_{g i}^{\min } \leq Q_{g i} \leq Q_{g i}^{\max } \quad i \in N_{\mathrm{G}}$

Transformer tap setting limit: $T_{m}^{\min } \leq T_{m} \leq T_{m}^{\max } \quad m \in N_{T}$

Line flow limits: $S_{l} \leq S_{l}^{\max } \quad l \in N_{l}$ 


Reactive power injected by FACTS controller limit: $-100 \leq \mathrm{Q}_{\mathrm{FACTS}, i} \leq 100$
$i \in \mathrm{N}_{\mathrm{FACTS}}$
$\begin{aligned} & \text { Reactance of the FACTS device limit: }-0.7 X \leq \mathrm{X}_{\mathrm{FACTS}, \mathrm{i}} \leq 0.2 \mathrm{X} \\ & i \in \mathrm{N}_{\mathrm{FACTS}}\end{aligned}$

Where,

$N_{P Q} \quad$ Total number of load buses

$V_{L}, I_{L} \quad$ voltages and currents of the load buses

$V_{G} I_{G} \quad$ Voltages and currents of Generator buses

NG Total number of Generators

$P_{G i} \quad$ Injected active power at Bus i

$P_{D i} \quad$ Active power demand at bus i

$B_{i j} \quad$ Suseptance between bus $\mathrm{i}$ and $\mathrm{j}$

$N_{B} \quad$ Total number of buses

$Q_{G i} \quad$ Injected reactive power at bus $\mathrm{i}$

$Q_{D i} \quad$ Reactive power demand at bus i

k (i.j), $\quad i \in N_{B}, j \in N_{i}$

$N_{T} \quad$ Set of number of transformer branches

$S_{l} \quad$ Power flow in branch 1

The amount of reactive power compensation at each bus is represented by an unknown vector $\mathrm{B}$ has been used as a decision vector. The solution of the problem has a set of decision vectors $\mathrm{B}$ for which the corresponding function can be optimized in any dimension. The set of decision vectors is called as Pareto- optimal (P). The corresponding objective vectors (F) calculated confirm a set of known Pareto-optimal Front (PF).

\section{Modeling of FACTS controllers}

The models of SVC, TCSC and UPFC are given below:

A Model of SVC:

SVC is a shunt connected static Var generator which can be used for both capacitive and inductive compensation. SVC is considered as an ideal reactive power controller at bus $i$.

The change in reactive power at bus $i, \Delta Q_{i}=Q_{S V C}$

$B$ Model of TCSC:

TCSC is a series compensation device which consists of a series capacitor bank shunted by a thyristor controlled reactor. The overall transmission line effective series impedance is decreased or increased by adding a capacitive or inductive reactive. TCSC is modeled as a variable reactance.

$$
\mathrm{X}_{\mathrm{ij}}=\mathrm{X}_{\text {line }}+\mathrm{X}_{\mathrm{TCSC}}
$$


Where, $\mathrm{X}_{\mathrm{ij}} \quad$ series reactance of the transmission line across buses I and $\mathrm{j}$ after compensation $\mathrm{X}_{\text {line }}$

Reactance of the transmission line

$\mathrm{X}_{\mathrm{TCSC}}$

Reactance of the TCSC

To avoid overcompensation the value of $\mathrm{X}_{\mathrm{TCSC}}$ is chosen between $-0.7 \mathrm{X}_{\text {line }}$ and 0.2 .

C Model of UPFC:

UPFC is used to provide the independent shunt and series compensation. It is a combination of Static Synchronous Compensator and Static Synchronous series compensator interconnected through a d.c link. It has two control parameters a voltage source inserted in series with the line and the current source connected in shunt with the line. It is able to absorb or generate real and reactive power outputs depending on the rating of UPFC.

\section{Solution for MORPO problem using stochastic algorithms}

The formulated MORPO problem is solved using EP, TS and PSO algorithms.

A. EP based algorithm for solving MORPO problem:

The various steps for solving Multi-objective Reactive Power Optimization problem using EP are as follow:

Step 1: The initial population is generated randomly within the feasible range such that the distributions of the initial trial parents are uniform.

Ipi = [Vi1, Vi2 ... ViNv; T1, T2...TiNT; Qi1, Qi2 ... QiNq1, $]$

Where, $\mathrm{i}=1,2 \ldots \mathrm{NP}$,

The initial population is selected such that

$$
\begin{aligned}
& \mathrm{Vij}=\mathrm{U}\left(V_{i j}^{\min }, V_{i j}^{\max }\right), \\
& \text { Tik }=\mathrm{U}\left(T_{i k}^{\min }, T_{i k}^{\max }\right) \text { and } \\
& \text { Qil }=\mathrm{U}\left({ }^{\min }, Q_{i l}^{\max }\right)
\end{aligned}
$$

where $\mathrm{u}(\mathrm{x}, \mathrm{y})$ denotes a uniform random variable between the limits $\mathrm{x}$ and $\mathrm{y}$.

Then for each individual of the population the power flow equations are solved by running the load flow by Newton Raphson method.Then the Fitness value is evaluated using (10) and the maximum fitness value is stored.

$$
f t=F+\Phi_{1} \sum_{j \varepsilon N v}\left(V_{i j}-V_{i j}^{\mathrm{min}}\right)^{2}+\Phi_{2} \sum_{k \varepsilon N_{T}}\left(T_{i k}-T_{i k}^{\mathrm{min}}\right)^{2}+\Phi_{3} \sum_{l \varepsilon N_{Q}}\left(Q_{i l}-Q_{i l}^{\mathrm{min}}\right)^{2}
$$

The values of the penalty factors $\Phi_{1}, \Phi_{2}$ and $\Phi_{3}$ are chosen such that there is any constraint violation then the fitness value corresponding to that parent will be ineffective.

Step 2 Mutation: The offspring population is generated from Nm parent individuals.

Offspring population, $I_{o i}^{m}=[$ Voi1 Voi2... Voij; Toil Toi2... Toik; Qoil Qoi2... Qoil];

$$
\mathrm{oi}=\mathrm{Np}+1, \mathrm{~Np}+2, \ldots \mathrm{Np}+\mathrm{Nm}
$$


The elements of $I_{o i}^{m}$ are generated as $V_{o i j}=V_{i j}+N\left(0, \sigma_{V_{i j}}^{2}\right) ; j=1,2, \ldots N_{v}$,
$T_{o i k}=T_{i k}+N\left(0, \sigma_{T_{i k}}^{2}\right) ; k=1,2, \ldots N_{T}$ and $Q_{o i l}=Q_{i l}+N\left(0, \sigma_{Q_{i l}}^{2}\right) ; l=1,2, \ldots N_{Q}$.

Where $\mathrm{N}(0, \sigma 2)$ is a normal random variable with mean zero and variance $\sigma 2$.

The variance decides the width of the normal distribution curve corresponding to each variables. The variance of each variable are computed using the equations below:

$$
\begin{aligned}
\sigma_{V_{i j}}^{2} & =\beta \frac{f t_{i}}{f t_{\max }}\left(V_{i j}^{\max }-V_{i j}^{\min }\right) \\
\sigma_{T_{k j}}^{2} & =\beta \frac{f t_{i}}{f t_{\max }}\left(T_{i k}^{\max }-T_{i k}^{\min }\right) \\
\sigma_{Q_{i j}}^{2} & =\beta \frac{f t_{i}}{f t_{\max }}\left(Q_{i l}^{\max }-Q_{i l}^{\min }\right)
\end{aligned}
$$

Where $\beta$ is the scaling factor.

Then the fitness values corresponding to each offspring are calculated.

Step3 : The Np parent trial vectors Iij and their corresponding offspring $I_{i j}^{\prime}$, contend to survive within the competing pool. After the competition, the $2 \mathrm{~Np}$ trial solutions are ranked in descending order based on the score obtained. The first $\mathrm{Np}$ trial solutions will survive and are transcribed along with their fitness functions. The first $\mathrm{Np}$ solutions were used for the next generation.

Step 4: The mutation, competition process are repeated until the maximum number of iterations is reached. The solution at the end of the maximum number of iteration is the optimal solution.

\section{B. Tabu Search based algorithm for solving MORPO problem:}

The various steps for solving Multi-objective Reactive Power Optimization problem using Tabu Search (TS) are as follow:

Step 1: The initialization and mutation process are similar to that of the initialization and mutation process as explained in EP algorithm. The initial parent population and offsprings from $\mathrm{Nm}$ parent populations are generated,

Step 2: An offspring population $I_{o i}^{r}$ of size $\mathrm{Nr}$ is generated from the parent population in the recombination process using the equation

$$
I_{o i}^{r}=[\text { Voil Voi2 } \ldots \text { Voij; Toil Toi2... Toik; Qoil Qoi2... Qoil]; oi= } \mathrm{Np}+\mathrm{Nm}+1, \mathrm{~Np}+\mathrm{Nm}+2
$$
$\ldots 2 \mathrm{~Np}$

The elements of $I_{o i}^{r}$ should be selected within the feasible range as $I_{o i}^{r}=$ Ipi1 $+\gamma$ (Ipi2 - Ipi1), Where $\gamma$ is the recombination factor and Ipi1 and Ipi2 are randomly selected parent individuals.

Step 4: Initially during the first iteration, $\mathrm{Nm}$ and $\mathrm{Nr}$ are half of the population of Population size $\mathrm{Np}$. Then during the forthcoming iterations value of $\mathrm{Nm}$ or $\mathrm{Nr}$ is increased or decreases correspondingly to the solution in the previous iteration. The value of $\mathrm{Nm}$ and $\mathrm{Nr}$ are changed such that $\mathrm{Nm}+\mathrm{Nr}$ is always equal to $\mathrm{Np}$.

Step 4:The Tabu List of size TLS comprises of a list of pre-elected size containing previous best

solutions. The sum of the distance

$$
D_{i}=\sum_{s=1}^{T L S}\left|I_{i}-I_{\text {tabus }}\right| ; i=1,2, . .2 N_{p}
$$

of each individual in the Tabu List is calculated. Each individual is then assigned with two ranks one based on the fitness and the other based on the distance. Then based on the two ranks a weight $\mathrm{Wi}$ is determined. 


$$
\mathrm{Wi}=\mathrm{RFi}+\alpha \mathrm{RDi}
$$

Where $\alpha$ is the distance weight factor;

RFi is the Rank based on fitness value and

$\mathrm{RDi}$ is the Rank based on Di

Then the individuals are arranged in descending order according to $\mathrm{Wi}$ and the first Np's are considered for the next iteration. The best individuals replace the worst individuals in the Tabu List along with their fitness value.

\section{PSO based algorithm for solving MORPO problem:}

The steps for solving Multi-objective Reactive Power optimization Problem using Particle swarm Optimization is explained below:

Step 1: Initially $\mathrm{n}$ numbers of particles are generated randomly with uniform probability within the search range. Then the fitness value of each particle is calculated by running the load flow using the Newton Raphson method. The values of Pbest and Gbest are calculated. Initially the velocities of the particles are set to zero.

Step 2: Then velocity of each element of a particle is calculated using the Pbest and Gbest values.

The jth element velocity of ith particle,

$$
v_{i, j}^{i t e r+1}=K\left(\begin{array}{l}
w \cdot v_{i, j}^{i t e r}+\varphi_{1} \cdot \text { rand }()\left(\text { pbest }-x_{i, j}^{i t e r}\right)+ \\
\varphi_{2} \cdot \text { rand }()\left(\text { gbest }-x_{i, j}^{i t e r}\right)
\end{array}\right)
$$

Where,

$$
\begin{array}{ll}
S_{i}^{\text {iter }} & \text { set of n particles at a time or iteration } \\
V_{i}^{\text {iter }} & \text { Particle velocity for an iteration } \\
v_{i, j}^{i t e r} & \text { velocity of the } i_{\text {th particle in }} j \text { th dimension. } \\
w & \text { Inertia weight } \\
\varphi_{1}, \varphi_{2} & \text { Acceleration constant } \\
\text { rand }() & \text { Function that generates uniform random number in the range }[0,1] \\
\mathrm{K} & \text { Constriction constant }
\end{array}
$$

The $\mathrm{K}$ is calculated as

$$
K=\frac{2}{\left|2-\varphi-\sqrt{\varphi^{2}-4 \varphi}\right|}
$$

Such that $\varphi=\varphi_{1}+\varphi_{2}$ and $\varphi>4$

The inertia weight $\mathrm{W}$ is calculated using

$$
w=w_{\max }-\frac{w_{\max }-w_{\min }}{\text { iter }_{\max }} \times \text { iter }
$$

Where,wmax and wmin are the upper and lower limits of the inertia weight.

Position Updation

Then the position of each particle is updated based on their velocities. The position of the jth element of the ith particle is given by

$$
x_{i, j}^{i t e r+1}=x_{i, j}^{i t e r}+v_{i, j}^{i t e r+1}
$$


Step 3: The fitness of all the particles are calculated using the updated position and velocity. In case of MORPO the Gbest is obtained by using the sigma method and the Pbest is obtained by the local dominancy concept.

The process of velocity updation, position updation, Updation of Pbest and Gbest are performed until the maximum number of iteration is reached. The Gbest at the maximum number of iteration is the optimal solution.

\section{Fuzzy Implemented Stochastic Algorithm for MORPO}

Incase of EP, TS and PSO algorithm it is found that the computation time is large and they require more number of iterations to reach the global optimal solution. So inorder to decrease the computation time and minimize the number of iterations Fuzzy Logic strategy is implemented over the EP, TS and PSO algorithms. The fuzzy logic strategy is implemented in the EP which leads to an amendment called as Fuzzy mutated Evolutionary Programming (FMEP) In the TS algorithm the fuzzy logic strategy is implemented in the mutation and recombination process and it leads to Fuzzy Guided Tabu Search (FGTS). In PSO algorithm Fuzzy Logic strategy is used to determine an adoptive inertia weight of the PSO algorithm which leads to an amendment called as Fuzzy PSO (FPSO).

\section{A. Fuzzy Logic Strategy in the Mutation process of EP and TS:}

The mutation process in the EP and TS algorithms are same. The values of the variance $\sigma 2$ depends on $f t_{i} / f t_{\max },\left(V_{i j}^{\max }-V_{i j}^{\min }\right)$ or $\left(T_{i k}^{\max }-T_{i k}^{\min }\right)$ or $\left(Q_{i l}^{\max }-Q_{i l}^{\min }\right)$ and the scaling factor $(\beta)$ The $f t_{i} / f t_{\max }$ has the major influence with the variance and if the value of it is small then the width of the normal distribution will be small. $\left(V_{i j}^{\max }-V_{i j}^{\min }\right)$ or $\left(T_{i k}^{\max }-T_{i k}^{\min }\right)$ or ( $\left.Q_{i l}^{\max }-Q_{i l}^{\min }\right)$ is the search range. The search range is constant throughout out the process. The $\beta$ influences the convergence. If the $\beta$ is small it leads to premature convergence. It was found that the realtion between the factors seems to be arbitrary and ambiguous. So a fuzzy logic strategy where the search criteria are not precisely bound would be more appropriate than a crisp relationship.

Steps involved in the Fuzzy implementation in mutation process:

Step1 : The inputs and outputs are decided. The inputs are fti/ftmax and $\left(V_{i j}^{\max }-V_{i j}^{\min }\right)$ or ( $\left.T_{i k}^{\max }-T_{i k}^{\min }\right)$ or $\left(Q_{i l}^{\max }-Q_{i l}^{\min }\right)$. The output of the fuzzy logic control is the variance $\sigma 2$ corresponding to the control variables.

Step 2: Fuzzification of input and output using triangular membership is done using five fuzzy linguistic sets.

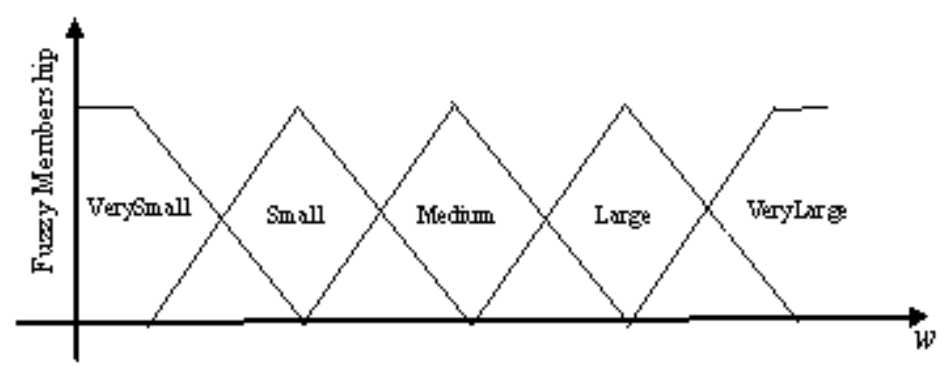

Step 3: The mutation scaling factor is resolved into the fuzzy control logic. Fuzzy rule base is formulated based on their ranges for all possible combinations. 
Table 1. Fuzzy Rule Base

\begin{tabular}{llllll}
\hline Input 1 & VerySmall & Small & Medium & Large & VeryLarge \\
\hline VerySmall & VerySmall & VerySmall & Small & Small & Small \\
\hline Small & VerySmall & Small & Small & Medium & Medium \\
\hline Medium & VerySmall & Small & Medium & Large & Large \\
\hline Large & Small & Medium & Large & VeryLarge & VeryLarge \\
\hline VeryLarge & Small & Medium & Large & VeryLarge & VeryLarge \\
\hline
\end{tabular}

Step 4: De-Fuzzification of output using centroid method is performed.

\section{B. Fuzzy Logic Strategy in Recombination of TS:}

In the recombination $\gamma$ is an arbitrary variable which deals with the search range of the parent population of the current iteration. The inputs to the fuzzy control are $I_{p i 2}-I_{p i 1}$ and $I_{p_{1}}-I_{T S}^{\min }$ and the output is the recombination factor $(\gamma)$. The steps involves in the implementation involves the selection of inputs and outputs, Fuzzification using five fuzzy linguistic sets, Formation of fuzzy rule base and the de-Fuzzification using centroid method as explained in the previous part.

\section{Fuzzy Logic strategy in the determination of inertia weight in PSO:}

Fuzzy logic strategy is used to determine an adoptive inertia weight. The inputs are $f t_{i} / f t_{\max }$ and

$$
\operatorname{Max}\left\{\left(V_{p, j}^{\max i}-V_{p, j}^{i t e r, i}\right) ;\left(V_{p, j}^{i t e r, i}-V_{p, j}^{\min t}\right)\right\}
$$

$$
\operatorname{Max}\left\{\left(T_{q, k}^{\max i}-T_{q, k}^{i t e r, i}\right) ;\left(T_{q, k}^{i t e r, i}-T_{q, k}^{\min t}\right)\right\}
$$

$\operatorname{Max}\left\{\left(Q_{r, l}^{\max i}-Q_{r, l}^{i t e r, i}\right) ;\left(Q_{r, l}^{i t e r, i}-Q_{r, l}^{\min t}\right)\right\}$

steps of the fuzzy logic implementation are similar to that of the previous sections with selection of input and outputs, Fuzzification, formation of fuzzy rules and de-Fuzzification.

\section{Simulation Results and Discussions}

Inorder to prove the feasibility and effectiveness of the proposed algorithms the standard IEE system is chosen as the test system. It consists of 6 generating units, 41 lines, 2 shunt reactors, 4 tap changing transformers and a total demand of $283.4 \mathrm{MW}$. The buses 2, 5, 8, 11 and 13 are the load buses and the remaining buses are the generator buses. Out of 41 lines the lines $(6,9),(6,10)$, $(4,12)$ and $(27,18)$ have the tap changing transformers and the reactive power sources are at buses 10 and 24. The algorithms were programmed in MATLAB V 7.1 installed in a Pentium IV, 2.5 $\mathrm{GHz}$ processor.

For the implementation of EP and Tabu search algorithm the population size is chosen as 30 and the scaling factor as 0.03 . The recombination and distance vector factors of TS algorithm are 0.04 and 0.01. In PSO algorithm the control parameters are chosen such that wmax,,as 0.9, wmin as 0.4,

$\varphi_{1}$ as 1.7 and $\varphi_{2}$ as 2.3. The penalty factors of the fitness function are chosen by trial and error method. Initially a small value is chosen. After the analysis if the constrained violated individuals have not been effectively eliminated, then the penalty factors will be increased until an acceptable solution is reached. The comparative convergence characteristics of EP, TS and PSO algorithm for MORPO are given in figure 1 . The convergence characteristics are drawn by treating the multiobjective problem as a single objective optimization problem by liner combination of the three objectives. The comparative results were given in Table 9. 
For the implementation of the FMEP and FGTS for MORPO problem the fuzzy logic data for the mutation process in EP and TS algorithm are given in table 2. The fuzzy logic data for the recombination process is given in table 3,4 and 5.The fuzzy logic data for the adoptive weight in PSO algorithm are given in Table 6, 7 and 8 .

Table 2. Fuzzy Logic Data for the Mutation process of EP and TS

\begin{tabular}{|c|c|c|c|c|c|c|c|}
\hline Fuzzy Set & $f t_{i} / f t_{\max }$ & & $\left(V_{i j}^{\max }-V_{i j}^{\min }\right)$ & $\left.T_{i k}^{\max }-T_{i k}^{\min }\right)$ & $\left(Q_{i l}^{\max }-Q_{i l}^{\min }\right)$ & $\beta$ & \\
\hline VerySmall & $\begin{array}{l}0.00001 \\
0.00004 \\
\end{array}$ & to & $0.95-0.98$ & $0.95-0.99$ & $-0.12--0.08$ & $\begin{array}{l}0.001 \\
0.005 \\
\end{array}$ & - \\
\hline Small & $\begin{array}{l}0.00003 \\
0.006 \\
\end{array}$ & to & 0.975- 0.99 & 0.97-0.99 & $-0.064--0.02$ & $\begin{array}{l}0.004 \\
0.06\end{array}$ & - \\
\hline Medium & 0.005 to 0.05 & & $0.985-1.0$ & $0.985-1.01$ & $-0.057-0.01$ & $\begin{array}{l}0.04 \\
0.08 \\
\end{array}$ & - \\
\hline Large & 0.03 to 0.5 & & 0.995-1.02 & $0.995-1.02$ & $0.09-0.15$ & $\begin{array}{l}0.075 \\
0.09 \\
\end{array}$ & - \\
\hline Verylarge & 0.4 to 1 & & $1.015-1.05$ & $1.015-1.1$ & $0.07-0.36$ & $\begin{array}{l}0.085 \\
0.1\end{array}$ & - \\
\hline
\end{tabular}

Table 3. Fuzzy Logic Data considering controllable voltage magnitude in the Recombination process

\begin{tabular}{llll}
\hline \multirow{2}{*}{ Fuzzy Set } & $I_{p i 2}-I_{p i 1}$ & $I_{p_{1}}-I_{T S}^{\min }$ & $\gamma$ \\
\hline VerySmall & $0.95-0.98$ & $0.95-0.98$ & 0.001 to 0.006 \\
\hline Small & $0.975-0.99$ & $0.975-0.99$ & 0.004 to 0.008 \\
\hline Medium & $0.985-1.0$ & $0.985-1.0$ & 0.07 to 0.09 \\
\hline Large & $0.995-1.02$ & $0.995-1.02$ & 0.085 to 0.2 \\
\hline VeryLarge & $1.015-1.05$ & $1.015-1.05$ & 0.15 to 0.3 \\
\hline
\end{tabular}

Table 4. Fuzzy Logic Data considering Tap changing transformer setting in the Recombination process

\begin{tabular}{llll}
\hline Fuzzy Set & $I_{p i 2}-I_{p i 1}$ & $I_{p_{1}}-I_{T S}^{\min }$ & $\gamma$ \\
\hline VerrySmall & $0.95-0.99$ & $0.95-0.99$ & 0.001 to 0.006 \\
\hline Small & $0.97-0.99$ & $0.97-0.99$ & 0.004 to 0.008 \\
\hline Medium & $0.985-1.01$ & $0.985-1.01$ & 0.07 to 0.09 \\
\hline Large & $0.995-1.02$ & $0.995-1.02$ & 0.085 to 0.2 \\
\hline Verylarge & $1.015-1.1$ & $1.015-1.1$ & 0.15 to 0.3 \\
\hline
\end{tabular}

Table 5. Fuzzy Logic Data considering Reactive Power Sources setting in the Recombination process

\begin{tabular}{llll}
\hline Fuzzy Set & $I_{p i 2}-I_{p i 1}$ & $I_{p_{1}}-I_{T S}^{\min }$ & $\gamma$ \\
\hline VerySmall & $-0.12--0.08$ & $-0.12--0.08$ & 0.001 to 0.006 \\
\hline Small & $-0.064--0.02$ & $-0.064--0.02$ & 0.004 to 0.008 \\
\hline Medium & $-0.057-0.01$ & $-0.057-0.01$ & 0.07 to 0.09 \\
\hline Large & $0.09-0.15$ & $0.09-0.15$ & 0.085 to 0.2 \\
\hline VeryLarge & $0.07-0.36$ & $0.07-0.36$ & 0.15 to 0.3 \\
\hline
\end{tabular}


Table 6. Fuzzy Logic data considering voltage magnitude for the adoptive weight

\begin{tabular}{|c|c|c|c|}
\hline Fuzzy Set & $f t_{i} / f t_{\max }$ & $\operatorname{Max}\left\{\left(V_{p, j}^{\max i}-V_{p, j}^{i t e r, i}\right) ;\left(V_{p, j}^{i t e r, i}-V_{p, j}^{\min t}\right)\right\}$ & $\mathrm{W}$ \\
\hline VerySmall & $\begin{array}{l}0.00001 \\
0.00004\end{array}$ & $0.95-0.98$ & $\begin{array}{l}0.001 \\
0.005\end{array}$ \\
\hline Small & 0.00003 to 0.006 & $0.975-0.99$ & $\begin{array}{ll}0.004 \quad- \\
0.06\end{array}$ \\
\hline Medium & 0.005 to 0.05 & $0.985-1.0$ & $0.04-0.08$ \\
\hline Large & 0.03 to 0.5 & $0.995-1.02$ & $\begin{array}{ll}0.075 & - \\
0.09 & \end{array}$ \\
\hline VeryLarge & 0.4 to 1 & $1.015-1.05$ & $0.085-0.1$ \\
\hline
\end{tabular}

Table 7. Fuzzy Logic data considering Tap changing transformers for the adoptive weight

\begin{tabular}{|c|c|c|c|}
\hline Fuzzy Set & $f t_{i} / f t_{\max }$ & $\operatorname{Max}\left\{\left(T_{q, k}^{\max i}-T_{q, k}^{i t e r, i}\right) ;\left(T_{q, k}^{i t e r, i}-T_{q, k}^{\min t}\right)\right\}$ & $\mathrm{w}$ \\
\hline \multirow[t]{2}{*}{ VerySmall } & 0.00001 & $0.95-0.99$ & 0.001 \\
\hline & 0.00004 & & 0.005 \\
\hline Small & 0.00003 to 0.006 & $0.97-0.99$ & $0.004-0.06$ \\
\hline Medium & 0.005 to 0.05 & $0.985-1.01$ & $0.04-0.08$ \\
\hline Large & 0.03 to 0.5 & $0.995-1.02$ & $0.075-0.09$ \\
\hline VeryLarge & 0.4 to 1 & $1.015-1.1$ & $0.085-0.1$ \\
\hline
\end{tabular}

Table 8. Fuzzy Logic data considering Reactive Power Sources for the adoptive weight

\begin{tabular}{|c|c|c|c|}
\hline Fuzzy Set & $f t_{i} / f t_{\max }$ & $\operatorname{Max}\left\{\left(Q_{r, l}^{\max i}-Q_{r, l}^{i t e r, i}\right) ;\left(Q_{r, l}^{i t e r, i}-Q_{r, l}^{\min t}\right)\right\}$ & w \\
\hline VerySmall & $\begin{array}{l}0.00001 \\
0.00004 \\
\end{array}$ & $-0.12--0.08$ & $\begin{array}{l}0.001 \\
0.005 \\
\end{array}$ \\
\hline Small & 0.00003 to 0.006 & $-0.064--0.02$ & $0.004-0.06$ \\
\hline Medium & 0.005 to 0.05 & $-0.057-0.01$ & $0.04-0.08$ \\
\hline Large & 0.03 to 0.5 & $0.09-0.15$ & $0.075-0.09$ \\
\hline VeryLarge & 0.4 to 1 & $0.07-0.36$ & $0.085-0.1$ \\
\hline
\end{tabular}

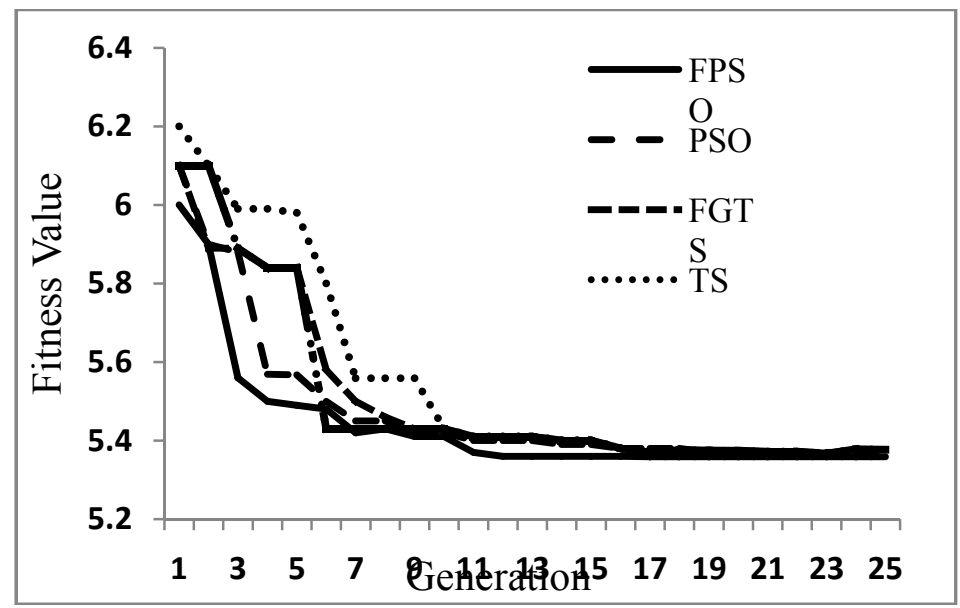

Figure 1. Convergence Characteristics of EP, FMEP, TS, FGTS, PSO and FPSO algorithms 


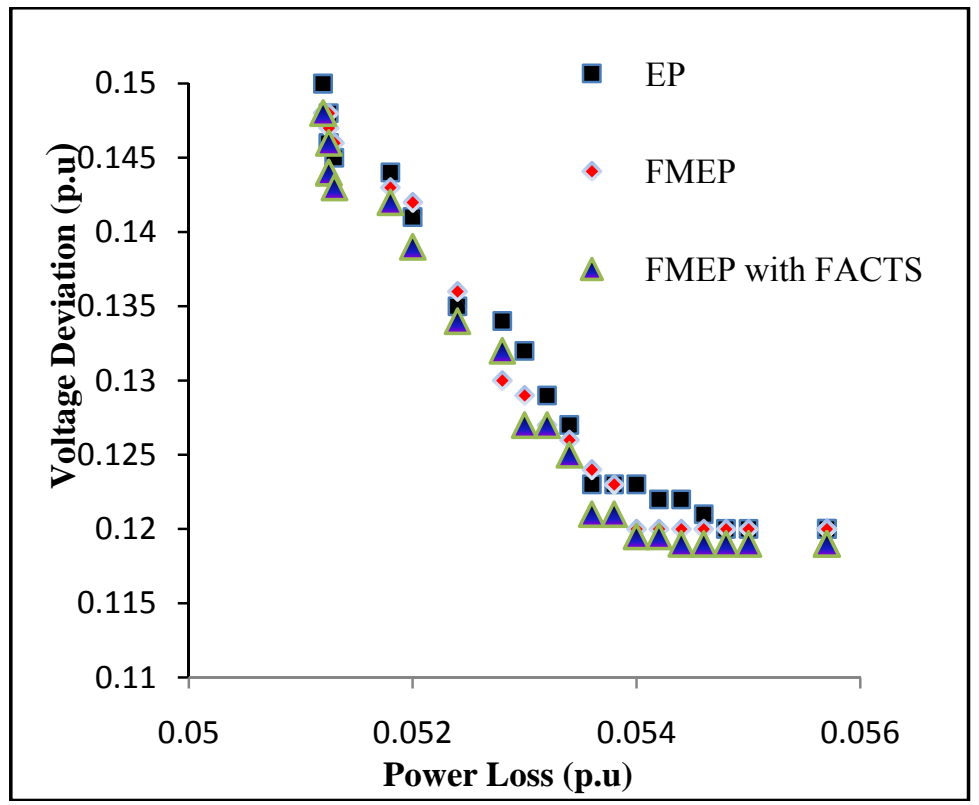

Figure 2. pareto set- Power Loss/ Voltage deviation of EP, FMEP and FMEP with FACTS controller (UPFC)

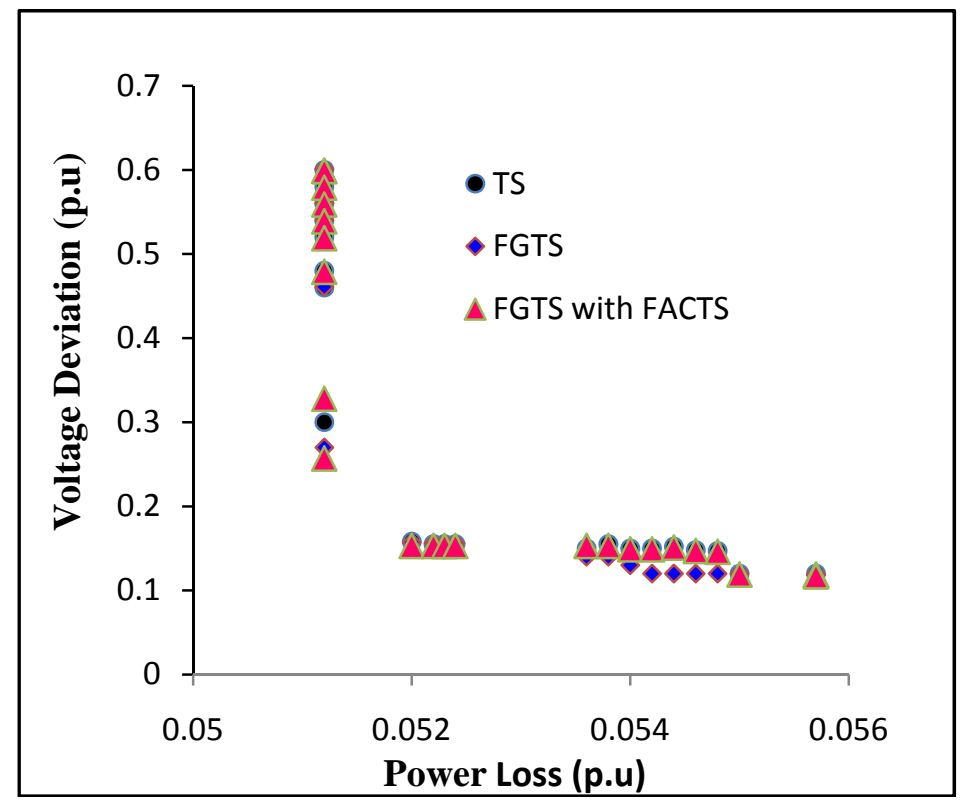

Figure 3. pareto set- Power Loss/ Voltage deviation of TS, FGTS and FGTS with FACTS controller (UPFC) 


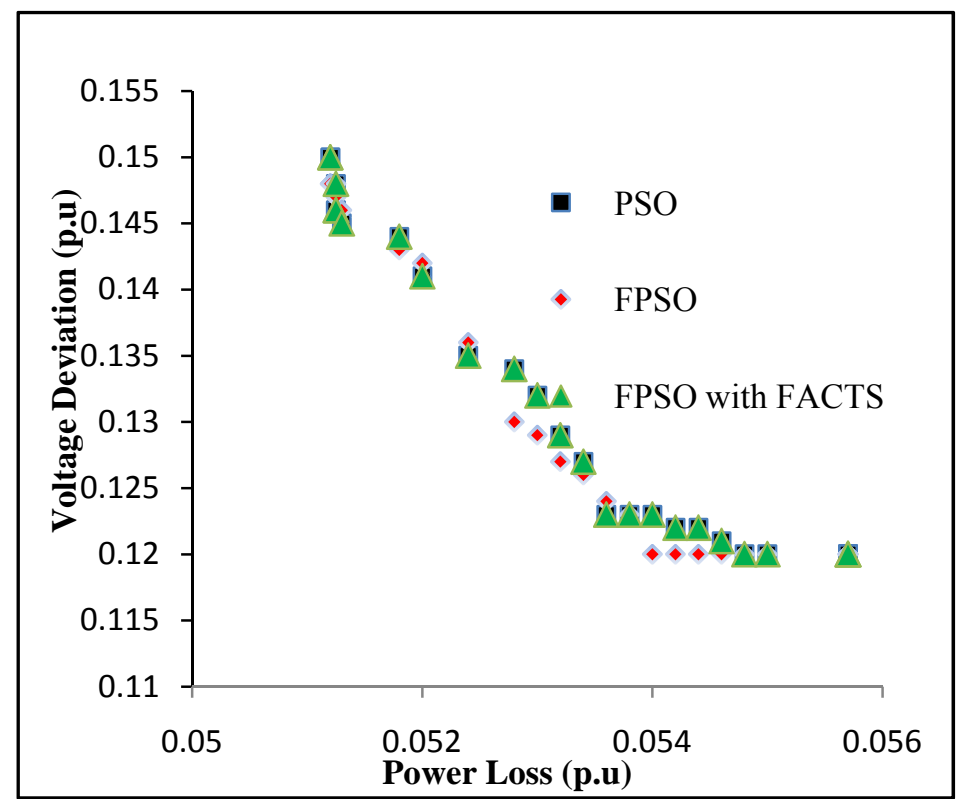

Figure 3. pareto set- Power Loss/ Voltage deviation of PSO, FPSO and FPSO with FACTS controller (UPFC)

The proposed algorithm of FMEP, FGTS and FPSO are coordinated with the FACTS devices like SVC, TCSC and UPFC. To coordinate the SVC for multi objective reactive power compensation, it is considered as the $\mathrm{P}-\mathrm{V}$ as the voltage generated by the $\mathrm{SVC}$ is independent of the system parameters. The SVC is located at bus 26 whose reactive power limits are 11.2 MVAr (injection) and -2.5 MVAr (absorption). The corresponding voltage limits are 0.9 and $1.1 \mathrm{p} . \mathrm{u} \mathrm{V}$. The SVC is located at bus 26 having the lower voltage magnitude of 0.9345 p.u (base case value). The comparative results of IEEE 30-bus test system corresponding to the proposed algorithms with SVC are given in Table.

Table 9. Comparative results of the proposed algorithm with and without SVC

\begin{tabular}{|c|c|c|c|c|c|c|c|c|c|}
\hline $\begin{array}{l}\text { Control } \\
\text { Variables }\end{array}$ & EP & $\begin{array}{l}\text { EP } \\
\text { (With } \\
\text { SVC) }\end{array}$ & $\begin{array}{l}\text { FMEP } \\
\text { (with } \\
\text { SVC) }\end{array}$ & $\mathrm{TS}$ & $\begin{array}{l}\text { TS } \\
\text { (With } \\
\text { SVC) }\end{array}$ & $\begin{array}{l}\text { FGTS } \\
\text { (with } \\
\text { SVC }\end{array}$ & PSO & $\begin{array}{l}\text { PSO } \\
\text { (With } \\
\text { SVC) }\end{array}$ & $\begin{array}{l}\text { FPSO } \\
\text { (With } \\
\text { PSO) }\end{array}$ \\
\hline V1 (p.u) & 1.009 & 1.05 & 1.05 & 1.05 & 1.05 & 1.05 & 1.016 & 1.049 & 1.05 \\
\hline V2 (p.u) & 1.006 & 1.0331 & 1.0262 & 1.036 & 1.0052 & 1.0265 & 1.012 & 1.037 & 1.0052 \\
\hline V5 (p.u) & 1.021 & 1.0011 & 0.9986 & 1.021 & 0.9506 & 1.0432 & 1.018 & 1.029 & 0.9506 \\
\hline V8 (p.u) & 0.998 & 1.014 & 1.0456 & 1.0325 & 0.973 & 1.0416 & 1.003 & 1.020 & 0.973 \\
\hline V11(p.u) & 1.066 & 1.013 & 1.098 & 0.986 & 1.0147 & 1.0172 & 1.061 & 1.002 & 1.0147 \\
\hline V13(p.u) & 1.051 & 1.0217 & 1.1 & 0.9945 & 1.0158 & 1.0865 & 1.051 & 0.995 & 1.0158 \\
\hline $\mathrm{T}(6-9)$ & 1.093 & 1.0092 & 1.0481 & 1.0368 & 1.0124 & 1.0335 & 1.093 & 1.002 & 1.0124 \\
\hline $\mathrm{T}(6-10)$ & 0.904 & 1.0163 & 0.9765 & 0.993 & 1.0286 & 1.0012 & 0.904 & 1.003 & 1.0286 \\
\hline $\mathrm{T}(4-12)$ & 1.002 & 0.9637 & 1.0497 & 1.03 & 0.95 & 0.9869 & 1.002 & 1.002 & 0.95 \\
\hline $\mathrm{T}(27-18)$ & 0.941 & 0.9865 & 1.05 & 0.948 & 0.9995 & 1.0226 & 0.941 & 1.039 & 0.9995 \\
\hline$\overline{\text { QSVC }}$ & - & 6.3241 & 6.004 & - & 6.246 & 6.121 & - & 6.250 & 6.004 \\
\hline $\mathrm{F} 1(\mathrm{Mw})$ & 5.889 & 5.32 & 5.244 & 5.53 & 5.545 & 5.32 & 5.386 & 4.996 & 4.555 \\
\hline F3 (p.u) & 0.1435 & 0.0017 & 0.0014 & 0.116 & 0.0018 & 0.0013 & 0.110 & 0.0133 & 0.125 \\
\hline
\end{tabular}


The TCSC is located in line 26 of IEEE 30-bus test system. The range of compensation by TCSC is 0 to $70 \%$ of line reactance. The optimal solution of IEEE 30-bus test system using the proposed algorithms with TCSC is presented in Table From Table it is inferred that with the inclusion of TCSC in line 26 the power loss has been decreased and the voltage deviation too.

Table 10. Comparative results of the proposed algorithm with TCSC

\begin{tabular}{lllllll}
\hline $\begin{array}{l}\text { Control } \\
\text { Variables }\end{array}$ & EP & FMEP & TS & FGTS & PSO & FPSO \\
\hline V1 (p.u) & 1.038 & 1.035 & 1.042 & 1.05 & 1.049 & 1.05 \\
\hline V2 (p.u) & 1.0262 & 1.0265 & 1.0052 & 1.0052 & 1.037 & 1.0265 \\
\hline V5 (p.u) & 0.9986 & 1.0432 & 0.9506 & 0.9506 & 1.029 & 1.0432 \\
\hline V8 (p.u) & 1.0456 & 1.0416 & 0.973 & 0.973 & 1.020 & 1.0416 \\
\hline V11(p.u) & 1.098 & 1.0172 & 1.0147 & 1.0147 & 1.0052 & 1.0172 \\
\hline V13(p.u) & 1.1 & 1.0865 & 1.0158 & 1.0158 & 0.9506 & 1.0865 \\
\hline $\mathrm{T}(6-9)$ & 1.0481 & 1.0335 & 1.0124 & 1.0124 & 0.973 & 1.0124 \\
\hline $\mathrm{T}(6-10)$ & 0.9765 & 0.7926 & 1.0286 & 1.0012 & 1.0147 & 1.0286 \\
\hline $\mathrm{T}(4-12)$ & 1.0497 & 1.0325 & 0.95 & 0.9869 & 1.0158 & 0.95 \\
\hline $\mathrm{T}(27-18)$ & 0.9865 & 1.05 & 0.9995 & 1.0226 & 1.0124 & 0.9995 \\
\hline $\mathrm{XTCSC}$ & -0.1385 & -0.1354 & -0.0745 & -0.1134 & -0.125 & 0.1352 \\
\hline F1(Mw) & 5.24 & 5.122 & 5.366 & 5.342 & 5.248 & 5.018 \\
\hline F3 (p.u) & 0.0152 & 0.0146 & 0.0195 & 0.0014 & 0.0177 & 0.122 \\
\hline
\end{tabular}

The UPFC is located in line 37 (connecting buses 25 and 26) of IEEE 30-bus system. The Series voltage coefficient $r$ limit values are 0 to 0.3 and the Series voltage angle $\gamma$ ranges from $-\pi$ to $\pi$ (radians) respectively. The results of the test system with UPFC are given in Table., Which tells the effectiveness of UPFC over other FACTS controllers. The power loss has minimized abruptly and the voltages at all the buses are within the limits.

Table 11. Comparative results of the proposed algorithm with UPFC

\begin{tabular}{lllllll}
\hline $\begin{array}{l}\text { Control } \\
\text { Variables }\end{array}$ & EP & FMEP & TS & FGTS & PSO & FPSO \\
\hline V1 (p.u) & 1.05 & 1.05 & 1.05 & 1.05 & 1.035 & 1.05 \\
\hline V2 (p.u) & 1.0351 & 1.0147 & 1.0348 & 1.0901 & 1.0265 & 1.0052 \\
\hline V5 (p.u) & 1.0029 & 0.9925 & 1.0214 & 0.9978 & 1.0432 & 0.9506 \\
\hline V8 (p.u) & 1.0347 & 1.0236 & 1.0489 & 0.9961 & 1.0416 & 0.973 \\
\hline V11(p.u) & 1.0340 & 1.0194 & 1.0891 & 1.0456 & 1.0172 & 1.0147 \\
\hline V13(p.u) & 1.0009 & 1.0125 & 1.0765 & 1.0786 & 1.0865 & 1.0158 \\
\hline T(6-9) & 1.0265 & 0.9912 & 1.0464 & 1.0025 & 1.0335 & 1.0124 \\
\hline T(6-10) & 0.9895 & 0.9956 & 1.05 & 0.9865 & 0.7926 & 1.0012 \\
\hline T(4-12) & 0.9941 & 1.0487 & 0.9976 & 1.05 & 1.0325 & 0.9869 \\
\hline T(27-18) & 1.0321 & 1.0025 & 0.9952 & 1.0487 & 1.05 & 1.0226 \\
\hline QSTATCOM & 0.1557 & 0.2126 & 0.0921 & 0.1554 & 0.1856 & 0.1753 \\
\hline r & 0.0845 & 0.0512 & 0.2432 & 0.0279 & 0.01685 & 0.0596 \\
\hline$\gamma$ in degree & 0.1802 & 0.1625 & 0.1445 & 0.1864 & 0.1375 & 0.1799 \\
\hline F1(Mw) & 4.7124 & 4.5984 & 4.6695 & 4.3589 & 4.5661 & 4.5266 \\
\hline F2 (p.u) & 0.020 & 0.0014 & 0.0015 & 0.0013 & 0.0018 & 0.0013 \\
\hline & & & & & &
\end{tabular}


The third objective is the minimization of the investment cost for compensation. With the compensation using the FACTS devices the UPFC gives an annual saving of 8,15,529.00 $\$$ and SVC and TCSC of 5,32,695.23 \$ and 7,96,685.52 \$ respectively. The Pareto Fronts of Power loss versus Voltage deviation for the proposed fuzzy based stochastic algorithms are given in figures 2, 3 and 4 which shows the effectiveness of the algorithm to generate Pareto solution in a well diverse manner. The Pareto with FACTS implies the better Pareto obtained using UPFC.

\section{Conclusion}

In this paper, an approaches based on Fuzzy based stochastic algorithms like FMEP, FGTS and FPSO are presented to solve the Multi-objective Reactive Power Optimization problem in coordination with the FACTS devices like SVC, TCSC and UPFC. The problem has been formulated as a Multi-objective optimization problem with non-linear competing objectives like active power loss, Voltage deviation and the investment cost of the compensation devices. The results obtained from the methods proved that they are efficient in solving the MORPO problem in coordination with the FACTS devices. Based on the observations in the coordination of FACTS devices with the network it was found that TCSC equilibrates the weakness of lines and transformers on their Suseptance whereas the SVC is suitable to reduce the line losses. The UPFC has better capability to minimize the losses and to give an important state of compensation.

\section{References}

[1] T Yalcinoz \& O Ko Ksoyo," A multi-objective optimization method to environmental economic dispatch", International Journal of Electrical power Energy Systems, Vol. 29, No. 1, pp. 42-50, 2007.

[2] Y. T. Hsaio, H. D. Chaing, C. C. Liu \& Y. L. Chen,” A Computer Package for Optimal Multi-objective VAR Planning in Large Scale Power Systems" , IEEE Transaction. on Power Systems, Vol. 9, No. 2, pp. 668-676, 1994.

[3] Y.L.Chen \& C.C. Liu, "Multi-objective VAr planning using the goal-attainment method", IEE Proceeding of Generation, Transmission and Distribution, Vol. 141, No. 3, pp. 227232,1994.

[4] Y.S.Brar,J.S.Dhillon \& D.P. Kothari," Multi-objective load dispatch by fuzzy logic based searching weightage pattern", Electrical Power System Research, Vol. 63, No. 2, pp. 149160,2002 .

[5] Y.L.Chen \& Y.L.Ke,” Multi-objective VAr planning for large-scale power systems using projection based two layer simulated annealing algorithms", IEE Proceeding of Generation, Transmission and Distribution, Vol. 151, No. 4, pp. 555-560, 2004.

[6] M.A. Abido, \& J.M Bakhashwain, "A Novel Multi- objective evolutionary algorithm, for optimal reactive power dispatch problem" , IEEE Transaction on Power Apparatus and Systems, pp. 1054-1057, 2003.

[7] J.E. Mendoza , D.A. Morales, R. A. Lopez,E.A. Lopez, J.C. Vannier \& C.A.C. Coellom, "Multi-objective location of automatic voltage regulators in a radial distribution network using a micro genetic algorithm", IEEE Transaction on Power System, Vol. 12, No. 1, pp. 404-412,2007.

[8] H. Alexandre , F Dias \& Jõao A. de Vasconcelos,"Multi-objective Genetic Algorithms Applied to Solve Optimization Problems", IEEE Transaction on Magnetics, Vol. 38, No. 2, pp. 1133-1136, 2002.

[9] M.A. Abido," A niched Pareto genetic algorithm for multiobjective environmental/economic dispatch", International Journal of Electric Power Energy Systems, Vol. 25, No. 2, pp. $97-$ $105,2003$.

[10] Abido M. A.," A niched Pareto genetic algorithm for multi objectives environmental /economic dispatch “, Electrical power and energy systems, pp. 97-105, 2003.

[11] M.A.Abido," Multiobjective Optimal VAR Dispatch Using Strength Pareto Evolutionary Algorithm," Proceedings of IEEE congress on Evolutionary Computation, pp. 730-736, 2006. 
[12] E Zitzler , M Laumanns ,\& L Thiele , "SPEA2: Improving the Strength Pareto Evolutionary Algorithm", TIK-Report 103, 2001.

[13] M.Varadarajan \&K.S. Swarup, " Solving multi-objective optimal power flow using differential evolution", IET Generation, Transmission \& Distribution, Vol. 2, No. 5, 720$730,2008$.

[14] Shil \& G Xu, "Self-adaptive evolutionary programming and its application to multi-objective optimal operation of power systems", Electrical Power System Research, Vol.57, No. 3, pp. 181-187, 2001.

[15] S.Durairaj, P.S.Kannan \& D.Devaraj, "Multi-objective VAR Dispatch using Particle Swarm Optimization”, International Journal of Emerging Electric Power Systems, Vol. 4, No. 1 (1082), pp. 1-16,2005.

[16] D. Gan, Z. Qu, \& H. Cai, "Large-Scale VAR Optimization and Planning by Tabu Search," Electric Power Systems Research, Vol. 39, pp. 195-204,1996.

[17] Mauriozio, Denna, Giancarlo Mauri, \& Anna Maria Zanaboni, "Learning fuzzy rules with Tabu-search an application to control" , IEEE Transactions on fuzzy systems, Vol. 7, No. 2, pp. 295-318, 1999.

[18] N.G. Hingorani \& L. Gyugyi, "Understanding FACTS: Concepts and Technology of Flexible AC Transmission Systems", IEEE Press, New York, 2000.

[19] W. Ongsakul \& P. Bhasaputra, "Optimal power flow with FACTS devices by hybrid TS/SA approach”, Electrical Power Energy Systems, Vol. 24, pp. 851-857, 2002.

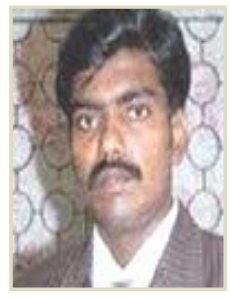

D. Silas Stephen received B.E degree in Electrical and Electronics Engineering from Manonmanium Sundaranar University in 2001 and M.E. degree in Power System Engineering from Anna University in 2004.At present he is pursuing his doctoral degree at Anna University, Chennai. He is now working as Assistant Professor at Panimalar Engineering College, Chennai. His research interests include Stochastic Algorithms on Power system optimization, FACTs and HVDC.

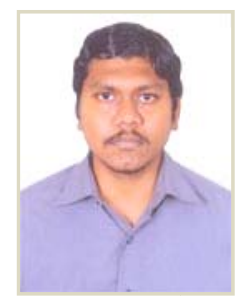

M. Devesh Raj received the B.E degree in Electrical and Electronics Engineering from Anna University in 2006 and received the M.E degree in Power Systems Engineering from College of Engineering Guindy Anna University Chennai in 2008. He is now working as Assistant Professor at SSN College of Engineering, Chennai. His research interests include Artificial Intelligence techniques on OPF, FACTs and HVDC.

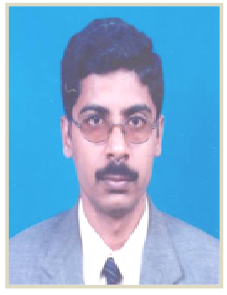

P. Somasundaram received the B.E. degree in Electrical and Electronics Engineering in 1998 from Madras University and obtained M.E. degree in power system engineering in 2000 from Annamalai University. He obtained his $\mathrm{Ph} . \mathrm{D}$. degree in power system engineering from Anna University Chennai, India in 2005. From 2000 to 2005, he worked as a Senior Lecturer in Department of Electrical and Electronics Engineering, College of Engineering Guindy Anna University Chennai. He is now an Assistant Professor in the Department of Electrical and Electronics Engineering at Anna University Chennai. His research interests include artificial intelligence techniques for power system problems, Reactive power optimization, congestion management and FACTs. 